\title{
Molecular characterization of Capra hircus lysosomal a-mannosidase and potential mutant site for the therapy of locoweed poisoning*
}

\author{
Kong Xiangya, Zhang Jiangye, Wu Ying, Li Jianfei and Li Qinfan ${ }^{凶}$ \\ College of Veterinary Medicine, Northwest A \& F University, Yangling 712100, Shaanxi, China
}

\begin{abstract}
Lysosomal a-Mannosidase (LAM) belongs to the glycoside hydrolyzing enzymes family 38 and is involved in the biosynthesis and turnover of $\mathrm{N}$-linked glycoproteins process. Locoweeds, which contain swainsonine (SW) that inhibits LAM, are the main poisoning plants in many regions of the world, and thereby resulting in animal poisoning or even death. Based on regions of protein sequence conservation between LAM from Bos taurus and Homo sapiens, we cloned CDNA encoding Capra hircus LAM (chLAM). Expression of CDNA in Pichia pastoris resulted in the secretion of aLAM activity into the culture medium. The recombinant chLAM was activated 1.6 and 1.2-fold with $\mathrm{Zn}^{2+}$ and $\mathrm{Ca}^{2+}$, respectively. By homology modeling, molecular docking and mutant analysis, we obtained the probable binding modes of SW at the allosteric sites of chLAM, and the potential mutant sites for the resistance to SW. Prediction of SW sensitivity to A28 W/G, D58 Y/G mutant chLAM is lower than wild type chLAM. The obtained results lead to a better understanding of not only interactions between substrate/SW and chLAM, but also of a potential strategy for a novel therapy for locoweed poisoning.
\end{abstract}

Key words: lysosomal a-mannosidase; Capra hircus; locoweed poisoning; molecular docking; Pichia pastoris expression

Received: 25 June, 2013; revised: 14 October, 2013; accepted: 16 March, 2014; available on-line: 21 March, 2014

\section{INTRODUCTION}

Glycoside hydrolyzing (GH) enzymes have been classified into over 100 families on the basis of sequence similarities (Henrissat \& Bairoch, 1993; Bourne \& Henrissat, 2001; Cantarel \& Coutinho, 2009). Lysosomal $\alpha$-mannosidase (LAM: EC 3.2.1.24) belongs to the sequence based $\mathrm{GH}$ family 38 (GH38) which are about 1000 residues long, and synthesized as a single polypeptide precursor, that is posttranslational cleaved into two to five polypeptide chains (Daniel et al., 1994). This cleavage pattern appears to vary in different organisms (Tollersrud et al., 1997). LAM is involved in N-glycan processing pathways and is associated with endoplasmic reticulum associated protein degradation process (Snaith, 1977; Uno et al., 2010). It is an exoglycosidase that hydrolyses all known $\alpha$ (axial)-mannosidic linkages on mannose-glycans originating from unfolded or endocytosed proteins (Snaith, 1975; Merkle et al., 1997; Athanasopoulos et al., 2005). LAM have been isolated and well studied from various animals, plants, and microorganisms sources, such as rat, jack bean, and Aspergillus phoenicis etc (Snaith, 1975; Snaith, 1977; Athanasopoulos et al., 2005). In humans, cattle, cat and guinea pig, the lack of LAM activity causes the autosomal recessive disease $\alpha$-mannosidosis (Michalski \& Klein, 1999). $\mathrm{Zn}^{2+}$ is known to activate LAM and has even been suggested for a therapy of some forms of human $\alpha$-mannosidosis (Bach et al., 1978).

Locoweeds are plants of the Astragalus and Oxytropis genera from North America, South America, and China that contain the indolizidine alkaloid swainsonine (SW) (van Kampen \& James, 1969). SW can inhibit LAM and Golgi mannosidase II (GAMII), which results in cellular vacuolation and degeneration similar to genetic $\alpha$-mannosidosis (Haraguchi et al., 2003; Barbosa et al., 2006). Poisoned animals appear neurological changes, reproductive disturbance, and emaciation. It causes many animal death and huge economical loss every year (Dantas et al., 2007). Nowadays, the main methods of preventing the locoweed poisoning are such as exterminating locoweed by artificial excavated, incineration and herbicides, which easily cause destruction of vegetation, and lead to desertification of grassland (Li, 2003). However, in the light of its ecological perspective, such as drought and cold resistance, strong reproductive capacity, high distribution of density and nutrition value, locoweed is an important forage resource $(\mathrm{Li}, 2003)$. Therefore, the prevention and cure of locoweed poisoning should focus on the comprehensive utilization.

Although many LAMs gene sequences from various organisms have been reported (Costanzi et al., 2006), there is no detailed research about the characteristic of locoweeds sensitivity animal Capra hircus LAM (chLAM). Many domestic and foreign scholars have made great progress on prevention and therapy of locoweed poisoning (Balogh et al., 1999), but the mechanism about SW inhabiting chLAM has not been revealed yet. The therapy for most $\alpha$-mannosidosis is providing normal enzyme to the lysosomal of abnormal cells (Ellinwood et al., 2004). The current approaches exist, or are under evaluation to deliver normal enzyme to abnormal cells by parenteral injection of purified recombinant enzyme (enzyme replacement therapy, ERT) (Gabrielli et al., 2010), bone

e-mail: liqinfan2012@aliyun.com

*The nucleotide sequence data has been submitted to DDBJ/ EMBL/GenBank databases under accession number JN602369. Abbreviations: AMA, a-mannosidase; LAM, Lysosomal AMA; chLAM, Capra hircus LAM; btLAM, Bos taurus LAM; hsLAM, Homo sapiens LAM; GAMII, Golgi mannosidase II; SW, swainsonine; GAPDH, glyceraldehyde-3-phosphate dehydrogenase; ERT, enzyme replacement therapy; pNP-a-Man, p-nitrophenyl a-mannoside; Man5, DMannose 5 
marrow transplantation (Wagner et al., 2010), and substrate reduction therapy (Marshall et al., 2010). Drawing lessons from $\alpha$-mannosidosis, to find out the mechanisms about SW inhabiting chLAM and to obtain a mutant chLAM which resistance to SW, appears very important.

Therefore, in this study, we described cDNA expression and molecular characterization of chLAM using Pichia pastoris as expression system. Using the technology of fluorescence quantitative PCR, chLAM gene expression profiling was obtained. Moreover, we generated a molecular 3-D model from the deuced amino acid sequence information using SWISS-MODEL web server by homology modeling program, which showed that the amino acid residues located at the active site of $c b \mathrm{LAM}$ are conserved between different species' LAM. Molecular docking method was also performed for further prediction on the structure properties, the probable binding modes of SW at the allosteric sites of $c b \mathrm{LAM}$, and the potential mutant sites for the resistance to SW. All these methods applied on $c h$ LAM could not only help understanding of the ligand-receptor interactions, but may be also applied to the prevention and for the therapy of locoweed poisoning.

\section{MATERIALS AND METHODS}

Materials. pNP- $\alpha-$ Man was from Sigma Co., St. Louis, U.S.A. Restriction enzymes were purchased from New England BioLabs Beverly, MA, USA. All synthetic oligonucleotide primers and Taq polymerase used in this study were from Takara Bio Inc., Otsu, Japan. PCR TA cloning kit, RNA isolation kit, and the Pichia expression kit were from Invitrogen San. Diego, CA, USA. Experimental animals, nanny goat, supplied by Laboratory Animal Center of Northwest A\&F University (Yangling, Shaanxi 712100, P. R. China). All other reagents were at least reagent grade and obtained from standard suppliers.

Nanny goat cDNA synthesis. Total RNA was isolated from ten 3-month-old nanny goats by the Trizol protocol (Invitrogen) and quantified by determining its absorbance at OD260/OD280. The animals were conducted according to institutional and ethical guidelines involving use of animals and the liver was quickly removed, freezed in liquid $\mathrm{N}_{2}$ and stored at $-80^{\circ} \mathrm{C}$. Poly (A) ${ }^{+}$RNA was isolated using Oligotex-dT30 (Takara, Kyoto, Japan) in accordance with the manufacturer's protocol. Total RNA $(2 \mu \mathrm{g})$ was reverse transcribed with M-MLV reverse transcriptase (Life Technologies, Inc.) using the manufacturer's protocols.

Polymerase chain reaction (PCR) and subcloning of PCR product. Degenerate oligonucleotide primers (F 5'-CTGCAGAGCCATGGT'TGGTG-3' and R 5'-CGGTAGTGACAT T'TAAATGGCT'T'TC-3') were designed based on Bos taurus LAM (btLAM, GenBank ID NM_174561.2) (Tollersrud et al., 1997). The amplification of goat liver LAM cDNA was carried out using PrimeSTAR-HS DNA polymerase according to the manufacturer's protocols. The sample were placed in a DNA thermal cycle programmed for temperature-step cycle of $92^{\circ} \mathrm{C}(45 \mathrm{~s}), 55^{\circ} \mathrm{C}(45 \mathrm{~s})$, and $72^{\circ} \mathrm{C}$ (3 min) for $30 \mathrm{cy}-$ cles. After the final cycle, the reaction was maintained at $72^{\circ} \mathrm{C}$ for $10 \mathrm{~min}$. The final reaction products were resolved on a $1 \%$ agarose gel containing ethidium bromide $(0.5 \mu \mathrm{g} / \mathrm{ml})$. Amplification products were purified from the gel using the DNA purification kit (Marathon, Clontech, Palo Alto, CA) and subcloned into pMD19-T sim- ple vector. Recombinant plasmids were isolated from liquid bacterial cultures and subjected to DNA sequencing.

Transformation of Pichia pastoris. chLAM cDNA was amplified by PCR using the oligonucleotide primers, 5'-GGCTGAAGCTGAAT'TCATGGCGGGATACAAGACATGCCC-3' (sense primer) and 5'-AAAGCTGGCGGCCGCCGCGGCGCCGTCCTCTTCCCATTGGA-3' (antisense primer). EcoRI and SacII restriction sites were designed into the sense and antisense primer, respectively. The primers permit the directional cloning of the amplified DNA in frame with the $\alpha$-factor leader sequence in $\mathrm{pPICZ} \alpha \mathrm{A}$ expression vector. The recombinant vector was linearized by Pme I and introduced into the Pichia host strain, X-33, using transformation procedures described in the Pichia expression manua (Hossain et al., 2010). pPICZ $\alpha$ A plasmid without an insert was conducted as negative controls. Positive transformants were isolated by zeocin ${ }^{\mathrm{TM}}$ screening (Hossain et al., 2010). After methanol induction, the clarified culture medium from each of the transformants was collected by centrifugation at $5000 \mathrm{r} / \mathrm{min}$ for $5 \mathrm{~min}$ and assayed for LAM enzyme activity

Assay system for LAM activity. Enzyme activity was measured by the chromogenic substrate $\mathrm{pNP}-\alpha-\mathrm{Man}$ (Liao et al., 1996). The incubation mixture contained 50 $\mu \mathrm{l}$ of sample, $50 \mu \mathrm{l}$ of $0.5 \mathrm{M}$ acetic acid/ $\mathrm{NaOH}$ buffer $(\mathrm{pH} 4.5)$, and $20 \mu \mathrm{l}$ of $10 \mathrm{mM} \mathrm{pNP}-\alpha-M a n$. Incubation lasted $90 \mathrm{~min}$ at $37^{\circ} \mathrm{C}$ and was terminated by addition of $200 \mu \mathrm{l}$ of $0.4 \mathrm{M}$ glycine/ $\mathrm{NaOH}$ buffer ( $\mathrm{pH} 10.5$ ). The released p-nitrophenol was measured by taking absorbance at $405 \mathrm{~nm}$. One unit of enzyme activity was defined as releasing $1 \mathrm{nmol}$ of $\mathrm{p}$-nitrophenol in 1 second at $37^{\circ} \mathrm{C}$.

SDS-polyacrylamide gel electrophoresis (SDSPAGE). Recombinant chLAM was analyzed by SDSPAGE (7.5\% polyacrylamide) in $25 \mathrm{mM}$ Tris, $192 \mathrm{mM}$ glycine and $0.1 \%(\mathrm{w} / \mathrm{v})$ SDS buffer using slab gel apparatus. After electrophoresis, the gel was stained with Coomassie Brilliant Blue R-250.

Effects of $\mathrm{pH}$, temperature, and metal ions on the activity of $\boldsymbol{c h L A M}$. The effects of $\mathrm{pH}$ on the activity of recombinant $c h \mathrm{LAM}$ were determined using $\mathrm{pNP}-\alpha-$ Man as substrates at various $\mathrm{pH}$ levels (3.0-8.0). The effects of temperature were also examined at various temperatures $\left(20-80^{\circ} \mathrm{C}\right)$ followed by standard assay method described in the earlier section. The enzymatic activities were calculated as percent $(\%)$ relative to the highest sample observed in this assay. The effects of metal ions on the activity of recombinant $c h \mathrm{LAM}$ were investigated using $\mathrm{Fe}^{2+}, \mathrm{Fe}^{3+}, \mathrm{Mn}^{2+}, \mathrm{Co}^{2+}, \mathrm{Ca}^{2+}, \mathrm{Zn}^{2+}, \mathrm{Cu}^{2+}, \mathrm{Mg}^{2+}$, $\mathrm{Al}^{3+}$, and EDTA at a concentration of $5 \mathrm{mM}$.

Real-time quantitative PCR (qPCR). qPCR is a method for rapid and reliable quantification of $\mathrm{mRNA}$ transcription. The glyceraldehyde-3-phosphate dehydrogenase (GAPDH) was used as an interior reference to normalise mRNA levels between different samples for an exact comparison of mRNA transcription level. The sequence of the primers for GAPDH gene was F 5' TGACCCTGGACT'TGACGAACR 3' and $\mathrm{R}$ 5' AAgGAgAAgGAtgGGGTGTG 3'. The primer sequence for chLAM gene was 5' GAGAAGGCTGGGGCTCACT'TR 3' and R 5' GT'TCACGCCCATCACAAACA 3'. PCR reactions were performed on Bio-Rad iQ5 System. For each PCR run with SYBR Green I detection, a melting curve analysis was performed to guarantee the specificity in each reaction tube. The experimental procedure and calculation method was assayed according to Preter and coworkers (De Preter et al., 2002). 


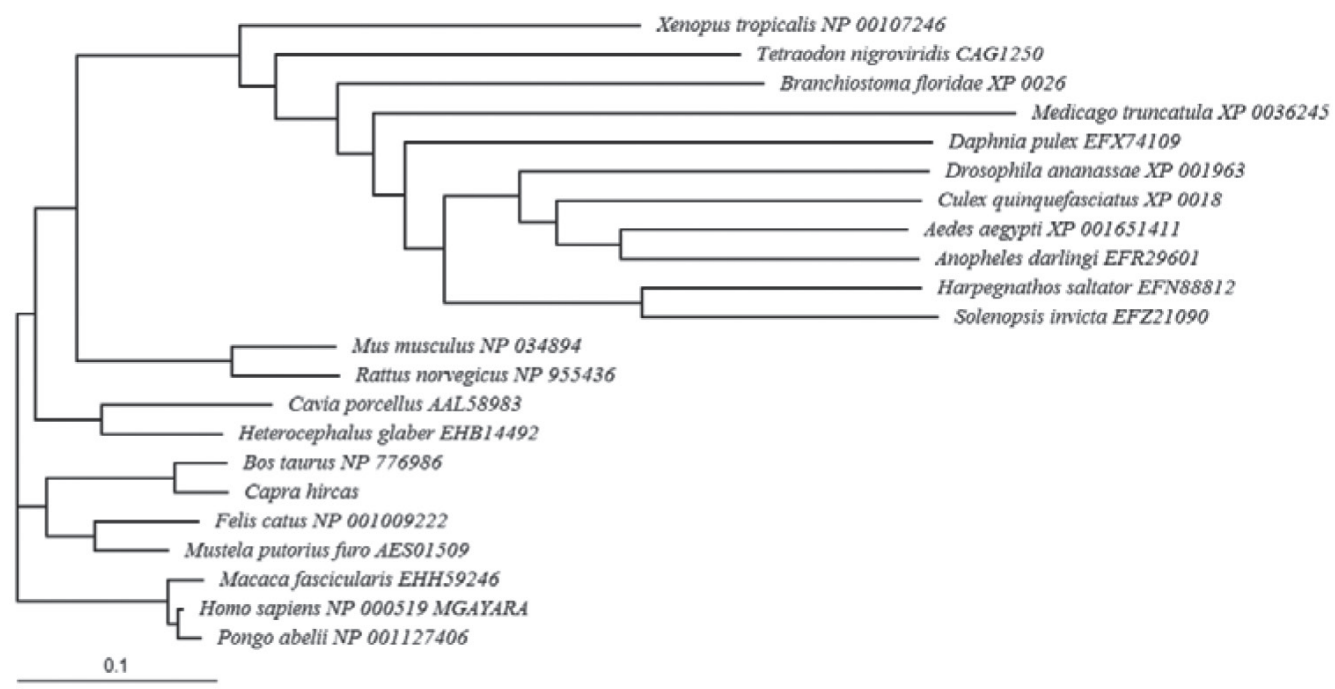

Figure 1. Phylogenetic tree of amino acid sequence from LAM homologues found in NCBI/Blastp search Multiple sequence alignment was performed using ClustalX 1.83 program. A total of 22 genes those characterized as GH38 were taken into consideration for the construction of phylogenetic tree. The analyzed chLAM is very closed to btLAM.

Bioinformatics analysis. The signal peptide was detected by SignalP 3.0 Server (Petersen et al., 2011). ExPASy Proteomics Server (Gasteiger et al., 2003) was used to determine the isoelectric point $(\mathrm{pI})$ and theoretical molecular mass of chLAM. The secondary structure and $\mathrm{N}$-glycosylation sites in the deduced residue sequence of $c h$ LAM were identified by PredictProtein online software (Rost et al., 2004). The most conserved domains of chLAM were identified by conserved domain database search at the National Center for Biotechnology Information (NCBI) web server (Bauer \& Bryant, 2004). The phylogenetic reconstruction of chLAM and homologues sequences was carried out by ClustalX 1.83 (Thompson et al., 1997).

Homology modeling and docking analysis. 3D protein structure is an important source of information for better understanding the function of a protein. The 3D structure of $c h$ LAM was created by SWISS-MODEL server 3.5 (Kiefer et al., 2009). The template protein btLAM (PDB ID 1O7D chains A, B, C, D and E resolution of $2.7 \AA$ ) from Brookhaven Protein Database (http://www.pdb.org/pdb/home/home.do) identified by Blast Search (http://www.ncbi.nlm.nih.gov/BLAS) was employed here for the construction of the target proteins (Heikinheimo et al., 2003). In addition, the quality of the modeled structure was assessed by the protein structure verification Procheck module of the structural analysis and verification on-line server (Kiefer et al., 2009). In order to locate the appropriate binding orientations, and conformations of substrates (man5 and SW) into the enzyme (modeled wild type and mutant $c h$ LAM) binding pocket, molecular docking was carried out by AutoDock4.2. Detailed process can be easily available from other resources (Jiang et al., 2008).

\section{RESULTS}

\section{Cloning, sequencing and identification of chLAM}

We deposited the isolated nucleotide sequences which encoded $c h$ LAM by a PCR-based approach in the Gen-
Bank data base with accession number JN602369. The sequence of the longest clone contained a full length open reading frame as well as $10 \mathrm{bp}$ of 5'UTR and $68 \mathrm{bp}$ of 3'UTR sequences, respectively, based on a comparison with btLAM cDNA (Tollersrud et al., 1997).

\section{Sequence and phylogenetic analysis of chLAM}

A 3000 bp open reading frame (ORF) was obtained from cDNA sequence isolated from nanny goat. It coded for a polypeptide of 999 amino acid residues of which the first N-terminal 50 amino acid residues form the signal sequence. Therefore, mature $c h \mathrm{LAM}$ is a polypeptide containing 949 amino acid residues with a predicted pI 8.01 and molecular mass of $107.56 \mathrm{kDa}$. Further analysis showed that mature $c h \mathrm{LAM}$ amino acid sequence contained nine $\mathrm{N}$ glycosylation sites. The positions of predicted N-glycosylation sites were 134, 347, 499, 755, and 919 in the deduced amino acid sequence.

The Class II $\alpha$-mannosidase (AMA), which was classified under glycosyl hydrolase family 38 (GH38), conserved GH38 N-terminal domain, AMA middle domain and GH38 C-terminal domain and can cleave $\alpha 1-2, \alpha 1-3$, and $\alpha 1-6$ glycosidic linkages of glycoproteins (Hossain et al., 2010). The conserved domain database search program in NCBI web server showed that $c h \mathrm{LAM}$ also has such three kinds of consecutive conserved domains in the deduced residue sequence. Therefore, these results demonstrated that chLAM belongs to GH38. To determine the evolutionary position of $c h \mathrm{LAM}$, a neighbor-joining phylogenetic tree was constructed (Fig. 1). We used the deduced amino acid sequence of $c h \mathrm{LAM}$ as Blastp input to explore the neighbors or homologous sequences. For the phylogenetic reconstruction, we included 22 sequences, which were previously functionally characterized as LAM. The resulting neighbor-joining tree suggests that $c b \mathrm{LAM}$ is very close to btLAM (NP 776986).

\section{Protein expression in Pichia pastoris and enzymatic characterization}

In order to demonstrate that chLAM cDNA encodes a functional LAM enzyme activity by expression in Pichia 


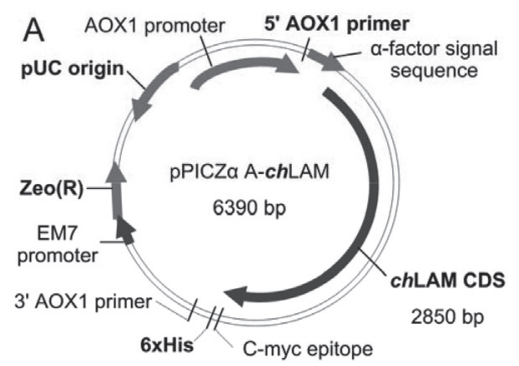

B

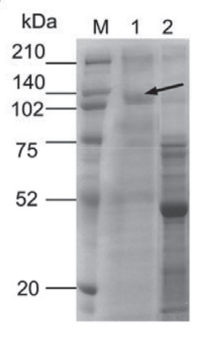

Figure 2. chLAM expression vector construct and SDS/PAGE

(A) ChLAM expression construct. chLAM CDNA (2850 bp) was expressed under AOX1 promoter with C-terminal His tag. (B) Purity recombinant Pichia pastoris expression product in X-33 was checked by $12 \%$ acrylamide and stained with Coomassie brilliant blue R-250. Lane M, Marker proteins; Lane 1, pPICZa A-chLAMX-33 (10 times concentrated); Lane 2, pPICZa A-X-33 (20 times concentrated). The proteins used in the calculation of molecular masses were Precision Plus ProteinTM Standards (BioRad): 210 $\mathrm{kDa}, 140 \mathrm{kDa}, 102 \mathrm{kDa}, 75 \mathrm{kDa}, 52 \mathrm{kDa}$ and $20 \mathrm{kDa}$.

pastoris, chLAM cDNA was subcloned into the Pichia expression vector. The signal peptide and 5\% 3 ' untranslated sequences of chLAM cDNA were truncated by PCR (Fig. 2A). The recombinant vector, $\mathrm{pPICZ} \alpha \mathrm{A}-c h \mathrm{LAM}$ was transformed into competent cells of E. coli JM109. The inserted DNA sequence was analyzed and further confirmed by PCR amplification and sequence analysis. The recombinant plasmid, $\mathrm{PPICZ} \alpha$ A-chLAM isolated and linearized with PmeI, was transformed into Pichia pastoris X-33 strain with zeocin ${ }^{\mathrm{TM}}$ screening. The expression of cDNA in this vector is driven by the methanolinducible promoter of the Pichia alcohol oxidase gene (AOX1). Growth of the cultures in media containing methanol as the sole carbon source results in high levels of expression, and secretion of the recombinant enzyme into the media. The resulting culture supernatant was analyzed for LAM activity by $p$-nitrophenyl- $\alpha-\mathrm{D}$ mannopyranoside (pNP- $\alpha$-Man) as a substrate. Using this enzyme assay system (Liao et al., 1996), high enzyme activity about $55 \mathrm{U}$ was found in the culture supernatant of Pichia with the recombinant plasmid $\mathrm{PPICZ} \alpha$ A-chLAM. In contrast, the extract from Pichia with the expression vector $\mathrm{PPICZ} \alpha \mathrm{A}$ (without $c h \mathrm{LAM} \mathrm{cDNA}$ insert) had no LAM activity. Compared to the predicted molecular mass of $107.56 \mathrm{kDa}$, the SDS-PAGE of the recombinant protein showed a major band at about 130 $\mathrm{kDa}$ (Fig. 2B), probably because of glycosylation.
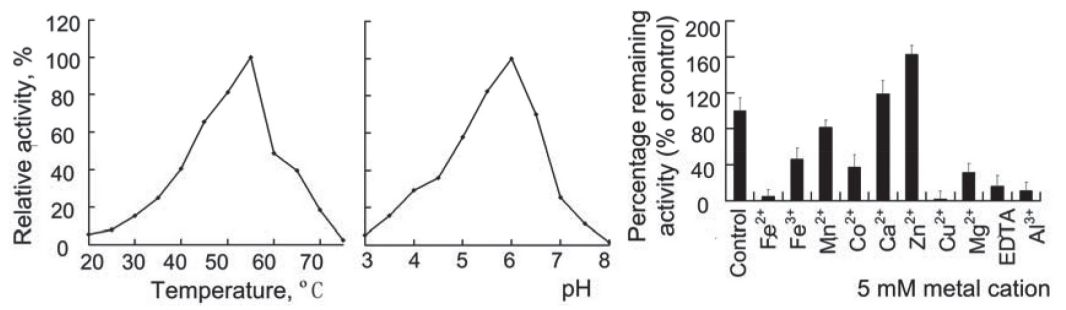

Figure 3. Effects of temperature, $\mathrm{pH}$ and metal cation on the activity of recombinant chLAM protein

(Left) Effects of temperature on the activity of chLAM. pNP-a-Man was used as substrates to analyze the effects of temperature on the activity of chLAM. chLAM was mixed with pNPa-Man in I mol/L acetic acid/NaOH buffer, $\mathrm{pH} 4.5$ and incubated at various temperatures $\left(20,30,40,50,60,70\right.$ and $\left.80^{\circ} \mathrm{C}\right)$. (Middle) The effects of $\mathrm{pH}$ on the activity of chLAM were examined using pNP-a-Man as substrates at various temperatures $\left(20-80^{\circ} \mathrm{C}\right)$ and $\mathrm{pH}$ levels (3.0-8.0). The buffers used were $0.1 \mathrm{M}$ Glycine- $\mathrm{HCl}(\mathrm{pH} 3.0), 0.1 \mathrm{M}$ Citrate $(\mathrm{pH} 3.5-5), 0.1 \mathrm{M}$ MES (pH5.5-6.5) and 0.1 M HEPES (pH7.0-8.0). (Right) The effects of metal ions on the activity of recombinant chLAM were investigated using $\mathrm{Fe}^{2+}, \mathrm{Fe}^{3+}, \mathrm{Mn}^{2+}, \mathrm{Co}^{2+}, \mathrm{Ca}^{2+}, \mathrm{Zn}^{2+}, \mathrm{Cu}^{2+}$, $\mathrm{Mg}^{2+}, \mathrm{Al}^{3+}$, and EDTA at a concentration of $5 \mathrm{mM}$.
Effects of $\mathrm{pH}$, temperature, and metal ions on the activity of chLAM

The effects of $\mathrm{pH}$ and temperature on the activity of $c h L A M$ were analyzed by $\mathrm{pNP}-\alpha-$ Man as substrates. ty rapidly declines after $\mathrm{pH} 6.0$ (Fig. 3), which suggests that $c h$ LAM could reside in the acidic region of cell. The of chLAM for substrate $\mathrm{pNP}-\alpha-$ was found to be at around $55^{\circ} \mathrm{C}$ (Fig. 3). The metal tivated 1.6 and 1.2 -fold with $\mathrm{Zn}^{2+}$ and $\mathrm{Ca}^{2+}$, respectively (Fig. 3).

\section{Tissue distribution of chLAM mRNA}

The expression patterns of chLAM gene in different tissues were shown by qPCR. At first, the design and pre-validation of the primers are essential to generate reliable data. As seen in Fig. $4 \mathrm{~A}$ and $4 \mathrm{~B}$, the dissociation curve appears as a single "stacked" peak at the amplicon Tm, suggesting the absence of other nonspecific products and primer dimers. For each of the 3 replicates of a sample, the average cycle time $(\mathrm{Ct})$, and the standard ev) were calculated. The relative expression for each sample was calculated by $2^{-\Delta \Delta \mathrm{Ct}}$ method. The reis plotted as a histogram graph (Fig. 4C). The results ous tissues, but at different levels. The greatest chLAM 列 script was less abundant in spleen, kidney, and heart.

\section{Molecular 3-D modeling}

An accurate three dimensional (3D) structure of the receptor is important for molecular docking (Ginalski, (D) the unavailability of $\mathrm{X}$-ray structure of chLAM, the homology modeling for the protein structure from its primary sequence was performed. The 1O7D) enzyme employed here is btLAM (PDB code resolution $(2.7 \AA)$. The initial sequence alignment of the target and template sequences was carried out using the ClustalW program, and the sequence identity obtained mode of the SWISS-MODEL web server was used to create the 3D structure of chLAM (Arnold et al., 2006).

The structural superposition of cbLAM model to the $\mathrm{X}$-ray crystal structure of btLAM indicating that the overall conformation of the modeling target is very similar to the template with RMSD $0.551 \AA$. The quality of the modeled structure was evaluated using structural assessment tool PROCHECK (Arnold et al., 2006). The total QMEAN-score (Composite scoring function for model quality estimation) of solved modeled structure of $c b$ LAM was 0.836 whereas btLAM had 0.864. The Z-score, 1.215 was also very close to the template structure 1.174 . Most importantly, the modeled $c h$ LAM structure had $99.1 \%$ of all its residues in conformationally permitted region as predicted by Procheck, and only a few residues were in the disallowed region. 


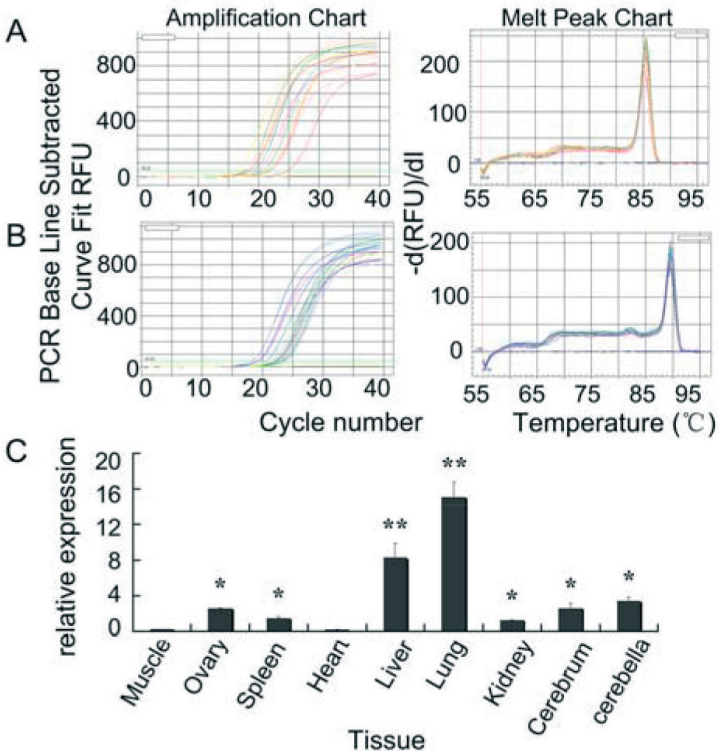

Figure 4. chLAM expression profiling

Dissociation and amplification curve of GAPDH (A) and chLAM (B) gene. $\mathrm{qPCR}$ results of $c h L A M$ gene relative expression in nine tissues. The relative mRNA expression level of chLAM gene in different tissues (C).

Therefore, an overall quality of modeled structure was good as checked by the program.

An overall structural model of chLAM included 949 residues with five structural parts, A (1-298 aa), B (299382 aa), C (383-541 aa), D (542-823 aa), and E (824-949 a). An important secondary structural motif comprised 20 helices and 42 strands (Fig. 5). The $\alpha$ and $\beta$ contents of the model protein were found to be $23.13 \%$ and $24.04 \%$, respectively, as predicted by the PredictProtein program.

\section{Docking and mutation analysis}

Figure $6 \mathrm{~A}$ and $6 \mathrm{C}$ show that D-Mannose 5 (man5) and SW nestles in the groove of wild type cbLAM characterized by residues His A23 (A-peptide histidine acid residue 23), Asp A25, Trp A28, Asp A147, Asp A270,

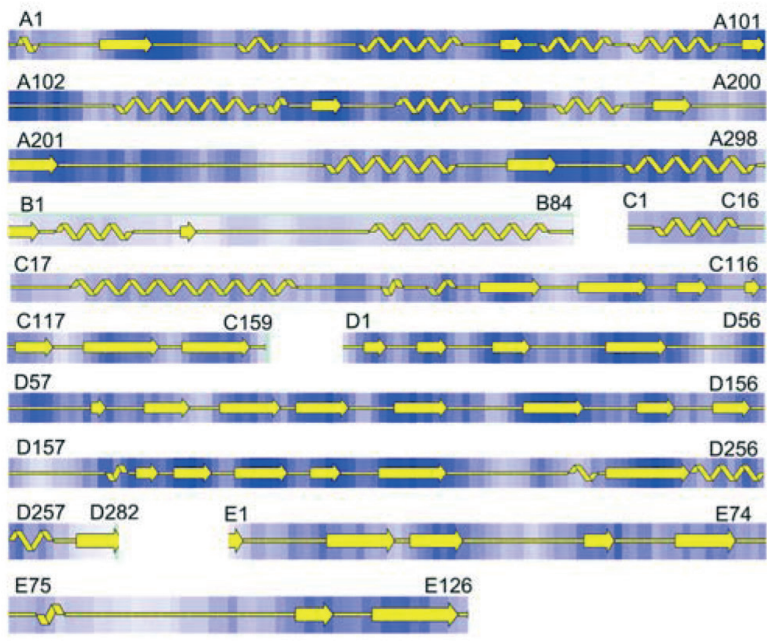

Figure 5. Prediction for secondary of chLAM

The secondary structural motif of chLAM comprises 20 a-helices and 42 strands. displays these nine key amino acids are conserved between different species' LAM.
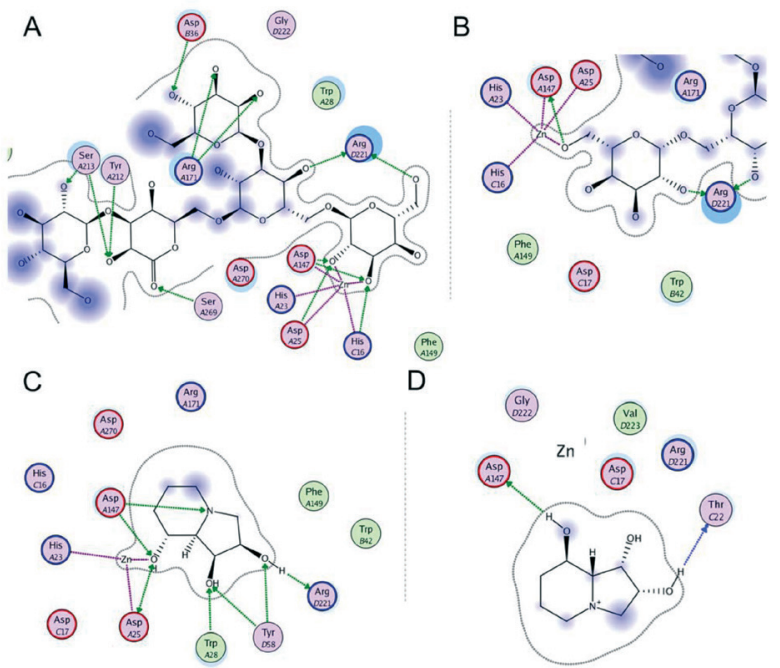

Figure 6. The molecular docked conformations of man5 (A/B) and SW (C/D) with wild type/mutant chLAM

His C16, Asp C17, Tyr D58, and Arg D221. Figure 7 displays these nine key amino acids are conserved between different species' LAM. The coordination bond formed between key residues and $\mathrm{Zn}^{2+}$, as well as $\mathrm{H}$ bond and hydrophobic interactions strongly stabilize the complexes, suggesting the potent binding of man5 and SW to wild type $c h L A M$. Figure $6 \mathrm{~A}$ depicts that man 5 is lodged in the active pocket surrounded by residues Asp A25, Asp A147, His C16, and Arg D221 via H-bonding and hydrophobic. Furthermore, it also forms a strong ionic bond with the catalytic $\mathrm{Zn}^{2+}$, which forms itself $\mathrm{H}$ bond with residue His A23, Asp A25, and His C16 of the pocket. Figure $6 \mathrm{C}$ reveals that $\mathrm{SW}$ is anchored in the binding site of wild type $c b \mathrm{LAM}$ via several $\mathrm{H}$-bonds by residues Asp A25, Trp A28, Asp A147, Tyr D58, and Arg D221. A $\mathrm{Zn}^{2+}$ also stabilizes the binding by mediating the interaction between SW and His A23, and Asp A25. Man5 and SW adopt a similar global conformation

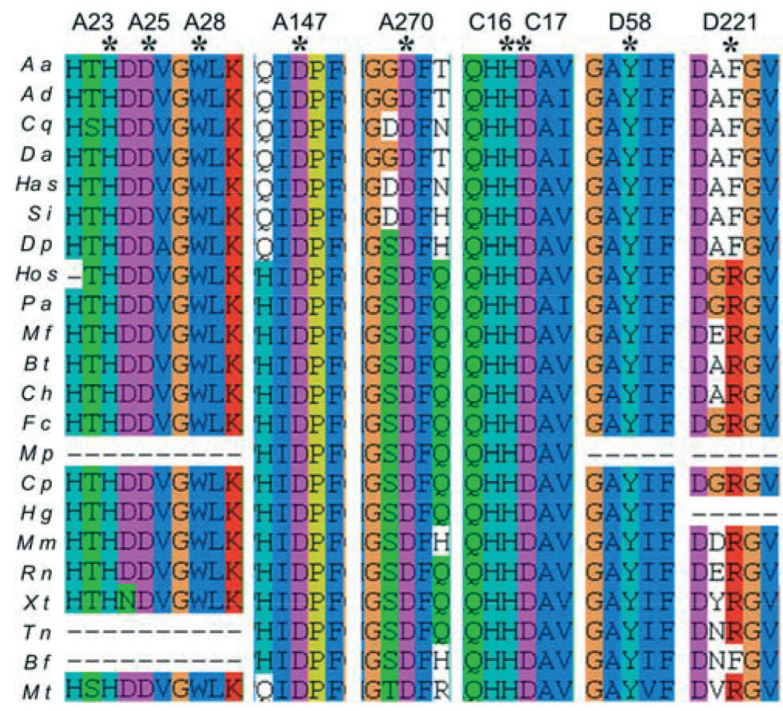

Figure 7. The alignments of key amino acid sequence of each species' LAM

Only key amino acids regions of 22 genes those characterized as GH38 are shown. *Represents the nine key amino acids which conserved between different species' LAM. 

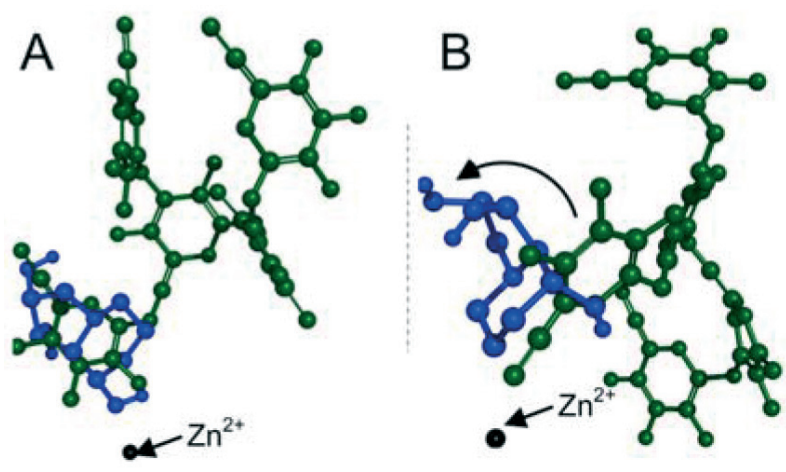

Figure 8. The superimposition of molecular docked conformations of man5 (green) and SW(blue) with wild type (A) and mutant (B) chLAM

binding to wild type chLAM, which explained why SW inhibited $c h \mathrm{LAM}$ function.

In order to find the mutation site for the therapy of locoweed poisoning, we closely inspect the difference binding modes of man5 and SW with the wild type chLAM. Overall, the interactions between man5/SW and His A23, Asp A25, Asp A147, and Arg D221 are conserved. However, two major differences were found: 1) man5 forms H-bond and ionic bond with His C16 of wild type chLAM, but SW is not; 2) Trp A28 and Tyr D58 of wild type chLAM are only seen as involved in $\mathrm{H}$ bond interaction with SW. If Trp A28 and Tyr D58 of wild type $c h$ LAM mutate to Gly, it may contribute to the resistance to SW.

Figure 8 shows the superimposition of molecular docked conformations of man5 and SW with wild type and $\mathrm{A} 28 \mathrm{~W} / \mathrm{G}$ and D58 Y/G mutant $c h \mathrm{LAM}$. Note that the mutant leads to a rotate nearly $100^{\circ}$, which makes SW stay $\sim 4 \AA$ away from the active site. The comparisons binding modes of the mutant $c h \mathrm{LAM}$ from docking studies are shown in Fig. 6B, 6D. Interestingly, man5 rotate $180^{\circ}$ as compared with that in wild type $c h$ LAM, but $\mathrm{H}$-bond number dose not appear to be significantly difference. For SW, H-bonds with His A23, Asp A25, Trp A28, Tyr D58, and Arg D221 are lost by A28 W/G and D58 Y/G mutants. Overall, A28 W/G and D58 Y/G mutation chLAM dose not appear to be significantly different of the wild type with man5, which nonetheless causes a shift to a lower binding affinity for SW. This could lead to a strategy for a novel therapy method in locoweed poisoning.

\section{DISCUSSION}

\section{Characterization of chLAM}

We have cloned and expressed chLAM cDNA with an intention to identify the molecular characterization and apply to the therapeutic strategies of locoweed poisoning through ERT or gene replacement approaches. Using pNP- $\alpha$-Man as substrates, high enzyme activity about 55 $\mathrm{U}$ was found in the culture supernatant of Pichia with the recombinant plasmid $\mathrm{pPICZ} \alpha$ A-chLAM. The recombinant $c h$ LAM shows optimum activity at $\mathrm{pH} 6.0$ and the activity rapidly declines after $\mathrm{pH}$ 6.0, which was consistent with other LAM (Kishimoto et al., 2001; Blom et al., 2008). The optimum temperature of recombinant $c b$ LAM was found to be at around $55^{\circ} \mathrm{C}$ (Fig. 3), which was slightly higher than those $\left(40-50^{\circ} \mathrm{C}\right)$ of other LAM
(Tollersrud et al., 1997). Recombinant chLAM is activated 1.6 and 1.2-fold with $\mathrm{Zn}^{2+}$ and $\mathrm{Ca}^{2+}$, respectively. For $\mathrm{Fe}^{2+}, \mathrm{Fe}^{3+}, \mathrm{Mn}^{2+}, \mathrm{Co}^{2+}, \mathrm{Cu}^{2+}, \mathrm{Mg}^{2+}, \mathrm{Al}^{3+}$, and EDTA, the activity of recombinant chLAM was found to be $4.4 \%$, $45.9 \%, 80.8 \%, 36.9 \%, 1.3 \%, 31.3 \%, 10.7 \%$, and $15.8 \%$ of the original. The effects of metal ions on the activity of $c h \mathrm{LAM}$ have similarities and also differences with other species' LAM. Such as $\mathrm{Zn}^{2+}$ are also required for the maximal activity of LAM from rat epididymis (Khan \& Basu, 1982). For LAM from human placental tissue, $\mathrm{Mn}^{2+}, \mathrm{Cu}^{2+}$, and $\mathrm{Co}^{2+}$ had no effect at $1 \mathrm{mM}$ concentration (Snaith \& Levvy, 1968).

For tissue specific expression profile analysis, $c h L A M$ gene is generally expressed in various tissues. Such as SW-induced lysosomal storage disease in goats, cytoplasmatic vacuolation was observed in the central nervous system and pancreatic acinar cells, epithelial cells of the kidney (Novikoff et al., 1985; Dantas et al., 2007). The highest levels of mRNA were observed in lung, and liver. But for mouse, tissues with greatest LAM mRNA abundance are epididymis, spleen and testis (Beccari et al., 1997). Results display that various species of LAM expression levels are not conserved.

\section{Mutant prediction for the treatment of locoweed toxicity}

Locoweeds, which contain the toxic indolizidine alkaloid SW that inhibits LAM, cause goats, horses, sheep poisoning, and sometimes even death, as well as large economical loss every year (van Kampen \& James, 1969). The action of $\alpha$-mannosidosis results in a lysosomal storage disorder which is caused by deficient activity of LAM closely mimics locoweeds poisoning, characterized by massive accumulation of mannose-containing oligosaccharides in affected individuals (Michalski \& Klein, 1999). Therapy for $\alpha$-mannosidosis usually dependens on providing normal enzyme to the lysosomal of abnormal cells (Ellinwood et al., 2004), such as heterologous bone marrow transplantation (Wagner et al., 2010), ERT (Gabrielli et al., 2010), and substrate reduction therapy (Marshall et al., 2010) et al. In this study, a potential therapy method for locoweed poisoning, which is based on $\alpha$-mannosidosis, is to deliver mutant LAM, that is lower or not inhibited by SW, to toxic animal.

Crawley et al. have used the guinea-pig model to investigate efficacy of ERT as a treatment for $\alpha$-mannosidosis (Crawley et al., 2006). Intravenous recombinant Homo sapiens LAM ( $h s$ LAM) was cleared by circulation with a half-life of $53 \mathrm{~h}$, with 1.4-fold of normal enzyme activity levels detected one week post-injection (Crawley et al., 2006). However, multiple injections of recombinant hsLAM in $\alpha$-mannosidosis guinea-pigs induced a very rapid humoral immune response precluding long-term intravenous treatment. In other studies, onset of ERT at birth in a feline mucopolysaccharidosis VI model using recombinant human protein resulted in lack of immune response compared with onset of therapy at a later age (Auclair et al., 2003). However, starting therapy at birth in the guinea-pig $\alpha$-mannosidosis model did not abrogate this immune response (Crawley et al., 2006). Further studies using recombinant guinea-pig $\alpha$-mannosidase would likely overcome immunological issues. Therefore, to obtain recombinant chLAM and find out a mutant chLAM which resistance to SW appears critically needed for goat locoweed toxicity.

Clearly, in vitro mutate remains a labor-intensive and time-consuming operation. Thus, more efficient and economical alternative methods should be employed, such 
as in silico molecular modeling approach that is used. Heikinheimo et al. have solved the structure of btLAM at $2.7 \AA$ resolution by molecular replacement (Heikinheimo et al., 2003). It opens the way to build realistic structural model of chLAM through in silico modeling and provides a major breakthrough to learn the binding interaction between man5/SW and chLAM. By docking analysis, we confirm that the activity site of chLAM is formed by nine key amino acids (His A23, Asp A25, Trp A28, Asp A147, Asp A270, His C16, Asp C17, Tyr D58, and Arg D221), which conserved between different species' LAM. Studies have shown that D221 R/H and R/W mutations are associated with $\alpha$-mannosidosis of cattle (Tollersrud et al., 1997) and guinea-pig (Berg et al., 1997), respectively.

After inspecting the difference binding modes of man5 and SW with the wild type chLAM, Trp A28 and Tyr D58 are only seen involved in H-bond interaction with SW. If Trp A28 and Tyr D58 of wild type $c h$ LAM mutate to Gly, it may contribute to the resistance to SW. Furthermore, it is no reported that this two amino acid mutations would affect the activity of LAM. The superimposition of molecular docked conformations of man5/SW with wild type and A28 W/G and D58 Y/G mutant $c b$ LAM show that SW stays $\sim 4 \AA$ away from the active site. H-bond number of man 5 dose not appear to be significantly different, but the $\mathrm{H}$-bonds with the His A23, Asp A25, Trp A28, Tyr D58, and Arg D221 are lost for SW. Overall, the mutation chLAM causes a shift to a lower binding affinity for SW, which nonetheless dose not appear to be significantly different of the wild type with man5.

ERT associated with A28 W/G and D58 Y/G mutant chLAM could lead to a strategy for a novel therapy method in locoweed poisoning. The next step is expressing the mutant $c b$ LAM with target characterizations. In another direction, the sequence of the mutant $c b \mathrm{LAM}$ can also be combined with transgenic technology or gene therapy.

\section{Acknowledgement}

The research is financially supported by the Fund of Northwest A \& F University.

\section{REFERENCES}

Arnold K, Bordoli L, Kopp J, Schwede T (2006) The SWISS-MODEL workspace: a web-based environment for protein structure homology modelling. Bioinformatics 22: 195-201.

Athanasopoulos VI, Niranjan K, Rastall RA (2005) The production, purification and characterisation of two novel mannosidases from Aspergillus phoenicis. Carbohyd Res 340: 609-617.

Auclair D, Hopwood JJ, Brooks DA, Lemontt JF, Crawley AC (2003) Replacement therapy in Mucopolysaccharidosis type VI: advantages of early onset of therapy, Mol Genet Metab 78: 163-174.

Bach G, Kohn G, Lasch EE, El Massri M, Ornoy A, Sekeles E, Legum C, Cohen MM (1978) A new variant of mannosidosis with increased residual enzymatic activity and mild clinical manifestation. Pediatr Res 12: 1010-1015.

Balogh KK, Dimande AP, van der Lugt JJ, Molyneux RJ, Naude TW, Welman WG (1999) A lysosomal storage disease induced by Ipomoed carnea in goats in Mozambique. I Vet Diagn Invest 11: 266-273.

Barbosa RC, Riet CF, Medeiros RMT, Lima EF, Barros SS, Gimeno EJ, Molyneux RJ, Gardner DR (2006) Intoxication by Ipomoea sericophylla and Ipomoea riedelii in goats in the state of Paraiba, Northeastern Brazil. Toxicon 47: 371-379.

Bauer AM, Bryant SH (2004) CD-Search: protein domain annotations on the fly. Nucleic Acids Res 32: 327-331.

Beccari T, Appolloni MG, Costanzi E, Stinchi S, Stirling JL, Fazia MAD, Servillo G, Viola MP, Orlacchio A (1997) Lysosomal alphamannosidases of mouse tissues: characteristics of the isoenzymes, and cloning and expression of a full-length cDNA. Biochem J 327 $45-49$.
Berg T, Tollersrud OK, Walkley SU, Siegel D, Nilssen O (1997) Purification of feline lysosomal alpha-mannosidase, determination of its cDNA sequence and identification of a mutation causing $\alpha$-mannosidosis in Persian cats. Biochem J 328: 863-870.

Blom H, Reyes F, Carlsson J (2008) Purification and characterization of an $\alpha$-mannosidase from the tropical fruit babaco. J Agri Food Chem 56: 10872-10887.

Bourne Y, Henrissat B (2001) Glycoside hydrolases and glycosyltransferases: families and functional modules. Curr Opin Struct Biol 11: 593-600.

Cantarel BL, Coutinho PM, Rancurel C, Bernard T, Lombard V, Henrissat B (2009) The Carbohydrate-Active EnZymes database (CAZy): an expert resource for Glycogenomics. Nucleic Acids Res 37: D233-D238.

Costanzi E, Balducci C, Cacan R, Duvetb S, Orlacchioc A, Beccari T (2006) Cloning and expression of mouse cytosolic $\alpha$-mannosidase (Man2c1). BBA-Gen Subjects 1760: 1580-1586.

Crawley AC, King B, Berg T, Meikle PJ, Hopwood JJ (2006) Enzyme replacement therapy in $\alpha$-mannosidosis guinea-pigs. Mol Genet Meta 89: 48-57.

Daniel PF, Winchester B, Warren CD (1994) Mammalian $\alpha$-mannosidase multiple forms but a common purpose. Glycobiology 4: 551-566.

Dantas AFM, Correaa FR, Gardner DR, Medeiros RMT, Barros SS, Anjos BL, Lucena RB (2007) Swainsonine-induced lysosomal storage disease in goats caused by the ingestion of Turbina cordata in Northeastern Brazil. Toxicon 9: 111-116.

De Preter K, Speleman F, Combaret V, Lunec J, Laureys G, Eussen BH, Francotte N, Board J, Pearson AD, De Paepe A, Van Roy N, Vandesompele J (2002) Quantification of MYCN, DDX1, and NAG gene copy number in neuroblastoma using a real-time quantitative PCR assay. Mod Pathol 15: 159-166.

Ellinwood NM, Vite CH, Haskins ME (2004) Gene therapy for lysosomal storage diseases: the lessons and promise of animal models. J Gene Med 6: 481-506.

Gabrielli O, Clarke LA, Bruni S, Coppa GV (2010) Enzyme-replacement therapy in a 5 -month-old boy with attenuated presymptomatic MPS I: 5-year follow-up. Pediatrics 125: 183-187.

Gasteiger E, Gattiker A, Hoogland C, Lvanyi I, Appel RD, Bairoch A (2003) ExPasy: the proteomics server for in-depth protein knowledge and analysis. Nucleic Acids Res 31: 3784-3788.

Ginalski K (2006) Comparative modeling for protein structure prediction. Curr Opin Struct Biol 16: 172-177.

Haraguchi M, Gorniak SL, Ikeda K, Minami H, Kato A, Watson AA, Nash R, Molyneux RJ, Asano N (2003) Alkaloidal components in the poisonous plant, Ipomoea carnea (Convolvulaceae). I Agric Food Chem 51: 4995-5000.

Henrissat B, Bairoch, A (1993) New families in the classification of glycosyl hydrolases based on amino acid sequence similarities. Biochem J 293: 781-788.

Heikinheimo P, Helland R, Leiros HKS, Ingar Leiros1, Karlsen S, Evjen G, Ravelli R, Schoehn G, Ruigrok R, Tollersrud OK, McSweeney S, Hough E (2003) The structure of bovine lysosomal alpha-mannosidase suggests a novel mechanism for low-pH activation. J Mol Biol 327: 631-644.

Hossain MA, Nakano R, Nakamura K, Hossain MT, Kimura Y (2010) Molecular characterization of plant acidic $\alpha$-mannosidase, a member of glycosylhydrolase family 38 , involved in the turnover of N-glycans during tomato fruit ripening. J Biochem 148: 603-616.

Jiang X, Kumar K, Hu X, Wallqvist A, Reifman J (2008) DOVIS 2.0: an efficient and easy to use parallel virtual screeningtool based on AutoDock 4.0. Chem Cent J 2: 1-7.

Khan FA, Basu D (1982) Isolation and characterization of lysosomal alpha-mannosidase of placental tissue. J Biosci 4: 133-138.

Kiefer F, Arnold K, Kunzli M, Bordoli L, Schwede T (2009) The SWISS-MODEL Repository and associated resources. Nucleic Acids Res 37: 387-392.

Kishimoto T, Hori H, Takano D, Nakano Y, Watanabe M, Mitsui T (2001) Rice $\alpha$-mannosidase digesting the high mannose glycopeptides of glutelin. Physiol Plantarum 112: 15-24.

Li JK (2003) The present situation and prospect of studies on locoweed in China. Scientia Agricultura Sinica 36: 1091-1099.

Liao YF, Lal A, Moremen KW (1996) Cloning, Expression, purification, and characterization of the human broad specificity lysosomal acid $\alpha$-mannosidase. The J Biol Chem 271: 28348-28358.

Marshall J, McEachern KA, Chuang WL, Hutto E, Siegel CS, Shayman JA, Grabowski GA, Scheule RK, Copeland DP, Cheng SH (2010) Improved management of lysosomal glucosylceramide levels in a mouse model of type I Gaucher disease using enzyme and substrate reduction therapy. I Inherit Metab Dis 33: 281-289.

Merkle RK, Zhang Y, Rues PJ, Liao YF, Moremen KW (1997) Cloning, expression, purification, and characterization of the murine lysosomal acid $\alpha$-mannosidase. BBA-Gen Subjects 1336: 132-146.

Michalski JC, Klein A (1999) Glycoprotein lysosomal storage disorders: $\alpha$ - and $\beta$-mannosidosis, fucosidosis and K-N-acetylgalactosaminidase deficiency. BBA-Mol Basis Dis 1455: 69-84. 
Novikoff PM, Touster O, Novikoff AB, Tulsiani DP (1985) Effects of swainsonine on rat liver and kidney: Biochemical and morphological studies. J Cell Biol 101: 339-349.

Petersen TN, Brunak S, Von HG, Nielsen H (2011) SignalP 4.0: discriminating signal peptides from transmembrane regions. Nat Methods 8: 785-786.

Rost B, Yachdav G, Liu J (2004) The PredictProtein server. Nucleic Acids Res 32: 321-326.

Snaith SM, Levvy GA (1968) $\alpha$-Mannosidase as a Zinc-dependent Enzyme. Nature 218: 91-92.

Snaith SM (1975) Characterization of jack-bean $\alpha$-D-mannosidase as zinc metalloenzyme. Biochem J 147: 83-90.

Snaith SM (1977) Multdple $\alpha$-mannosidase activities in mammalian tissues as shown by metal-ion activation. Biochem J 163: 557-564.

Thompson JD, Gibson TJ, Plewniak F (1997) The ClustalX windows interface: flexible strategies for multiple sequence alignment aided by quality analysis tools. Nucleic Acids Res 25: 4876-4882.
Tollersrud OK, Berg T, Healy P, Evjen G, Ramachandran U, Nilssen $\varnothing$ (1997) Purification of bovine lysosomal $\alpha$-mannosidase, characterization of its gene and determination of two mutations that cause $\alpha$-mannosidosis. Eur J Biochem 246: 410-419.

Uno Y, Hashidume S, Kurita O, Fujiwara T, Nomura K (2010) Dioscorea opposita Thunb. $\alpha$-mannosidase belongs to the glycosyl hydrolase family 38. Acta Physiol Plant 32: 713-718.

van Kampen KR, James LF (1969) Pathology of locoweed poisoning in sheep. Pathol Vet 6: 413-423.

Wagner JE, Yamamoto AI, McGrath JA, Hordinsky M, Keene DR, Woodley DT, Chen M, Riddle MJ, Osborn MJ, Lund T, Dolan M, Blazar BR, Tolar J (2010) Bone Marrow Transplantation for Recessive Dystrophic Epidermolysis Bullosa. New Engl J Med 363: 629-639. 